\title{
ON THE INNOVATION THEOREM
}

\author{
T. F. LIN
}

\begin{abstract}
Let $z(t), 0<t<T$, be the signal process and $y(t)=\int_{0}^{t} z(r) d r$ $+w(t)$ be the observation process where $w(t)$ is a process of independent increments. It is shown that, under certain conditions, the innovation process $v(t)=y(t)-\int_{0}^{t} E(z(r) \mid y(u), 0<u<r) d r$, has the same probability law as $w(t)$.
\end{abstract}

1. Introduction. Let $z(t), 0 \leqslant t \leqslant T$, be a $(t, \omega)$-measurable signal process in $R^{m}$ such that

$$
\int_{0}^{T} E|z(t)|^{2} d t<\infty
$$

where $|\cdot|$ denotes the $m$-dimensional vector norm as well as the absolute value of a number, and let

$$
y(t)=\int_{0}^{t} z(s) d s+w(t)
$$

for $0<t<T$, be the observation process where $w(t)$ is an $m$-dimensional Wiener process such that

$$
\begin{aligned}
& W(t)-w(s) \text { is independent of } \\
& \mathcal{G}_{s}=\sigma\{z(r), w(r) ; 0 \leqslant r \leqslant s\} \text { for } 0 \leqslant s \leqslant t \leqslant T .
\end{aligned}
$$

Let $\mathscr{F}_{s}=\sigma\{y(r): 0 \leqslant r \leqslant s\}, 0 \leqslant s \leqslant T$. Condition (1.1) implies the existence of $E\left\{z(s) \mid \mathscr{F}_{s}\right\}$ which is measurable in $(s, \omega)$. Indeed, this condition can be weakened somewhat (see [3, p. 21 and Remark, p. 23]). The process

$$
v(t)=y(t)-\int_{0}^{t} E\left\{z(s) \mid \mathscr{F}_{s}\right\} d s
$$

is called the innovation process. Application of the innovation processes to optimal filtering can be found in [1], [2], [4], [6], [7].

It has been known that $\left\{v(t), \mathscr{F}_{t}\right\}$ is an $L^{2}$-martingale with the same quadratic variation as $w(t)$. This result was extended to the case that $w(t)$ is an $L^{2}$-martingale with conditional independent increments w.r.t. $\left\{\mathcal{G}_{t}\right\}$ (see [5, Theorem 3]). If $w(t)$ is the Wiener process again, the continuity of $v(t)$

Received by the editors September 22, 1975.

AMS (MOS) subject classifications (1970). Primary 60J30; Secondary 60G35.

Key words and phrases. Process of independent increment, martingale, innovation process, observation process. 
implies that $v(t)$ is also a Wiener process (see [3, Lemma 2.2], [5, Theorem 2]). These results are the innovation theorems. Combining the above results, it is naturally interesting to know whether $v(t)$ has the same probability law as $w(t)$ if $w(t)$ is a process of independent increments. This paper shows that this is true, at least under certain conditions.

2. Generalization. Let all the notations be defined as in $\$ 1$ except that $w(t)$ is now a process of independent increments satisfying condition (1.2) and

$$
E w(t)=0, \quad E|w(t)-w(s)|^{2} \leqslant c^{2}(t-s),
$$

for $0 \leqslant s \leqslant t \leqslant T$ where $c \in R^{+}$. Let $x(t)=z(t)-E\left\{z(t) \mid \mathscr{F}_{t}\right\}, 0<t<$ $T$. Then

$$
v(t)=\int_{0}^{t} x(s) d s+w(t) .
$$

It is easy to see that $\left\{v(t), \mathscr{F}_{t}\right\}$ is a martingale.

For $0<s<t<T$, let $t_{k}=s+k(t-s) 2^{-n}, 0<k<2^{n}$. Also, for $0<k$ $<2^{n}$, let

$$
\Delta v\left(t_{k}\right)=v\left(t_{k+1}\right)-v\left(t_{k}\right), \quad \Delta w\left(t_{k}\right)=w\left(t_{k+1}\right)-w\left(t_{k}\right)
$$

and

$$
\Delta I_{k}(x)=\int_{t_{k}}^{t_{k+1}} x(r) d r .
$$

LEMMA 1. (a) $\lim _{n \rightarrow \infty} \Sigma_{k} E\left\{\left|\Delta I_{k}(x)\right|^{2}\right\}=0$.

(b) $\lim _{n \rightarrow \infty} \Sigma_{k} E\left\{\left|\Delta w\left(t_{k}\right)\right|\left|\Delta I_{k}(x)\right|\right\}=0$.

Proof. By Schwarz's inequality and Fubini's theorem,

$$
\begin{aligned}
E\left\{\left|\Delta I_{k}(x)\right|^{2}\right\} & \leqslant\left(t_{k+1}-t_{k}\right) \int_{t_{k}}^{t_{k+1}} E|x(s)|^{2} d s, \\
E\left\{\left|\Delta w\left(t_{k}\right)\right|\left|\Delta I_{k}(x)\right|\right\} & \leqslant\left\{E\left|\Delta w\left(t_{k}\right)\right|^{2} E\left|\Delta I_{k}(x)\right|^{2}\right\}^{1 / 2} \\
& \leqslant c\left(t_{k+1}-t_{k}\right)\left\{\int_{t_{k}}^{t_{k+1}} E|x(s)|^{2} d s\right\}^{1 / 2} .
\end{aligned}
$$

In deriving the last inequality, we have used both (2.1) and (2.2). Since

$$
E|x(s)|^{2} \leqslant E|x(s)|^{2}+E\left|E\left\{z(s) \mid \mathscr{F}_{s}\right\}\right|^{2}=E|z(s)|^{2},
$$

(a) follows from (2.2) and (1.1). From (2.3),

$$
\sum_{k} E\left\{\left|\Delta w\left(t_{k}\right)\right|\left|\Delta I_{k}(x)\right|\right\} \leqslant c(t-s) \max _{k}\left\{\int_{t_{k}}^{t_{k+1}} E|z(s)|^{2} d s\right\}^{1 / 2} .
$$

Therefore, (b) follows from the above inequality and (1.1).

For $0<k<2^{n}$, let

$$
f_{k}=e^{i \lambda \cdot\left[w(t)-w\left(t_{k}\right)+v\left(t_{k}\right)-v(s)\right]},
$$


where $\lambda \in R^{m}$ and where "." denotes the dot product in $R^{m}$.

LEMMA 2. For $0<k<2^{n}$,

$$
E\left(f_{k+1}-f_{k} \mid \mathscr{F}_{s}\right)=E\left\{f_{k} e^{-i \lambda \cdot \Delta w\left(t_{k}\right)}\left(J_{1}+J_{2}\right)(k) \mid \mathscr{F}_{s}\right\}
$$

with

$$
\left|J_{1}(k)\right|<\frac{1}{2}|\lambda|^{2}\left|\Delta I_{k}(x)\right|^{2}, \quad\left|J_{2}(k)\right|<|\lambda|^{2}\left|\Delta w\left(t_{k}\right)\right|\left|\Delta I_{k}(x)\right| .
$$

Proof. Note that if we write

$$
e^{i u}=1+R_{1}(u)=1+i u+R_{2}(u)
$$

then

$$
\left|R_{1}(u)\right| \leqslant|u|, \quad\left|R_{2}(u)\right| \leqslant \frac{1}{2}|u|^{2} .
$$

Now since $\Delta v\left(t_{k}\right)=\Delta I_{k}(x)+\Delta w\left(t_{k}\right)$, it is easy to check that

$$
f_{k+1}-f_{k}=f_{k}\left(e^{i \lambda \cdot \Delta I_{k}(x)}-1\right) \text {. }
$$

Therefore, by using (2.6),

$$
\begin{aligned}
& E\left(f_{k+1}-f_{k} \mid \mathscr{F}_{s}\right)=E\left\{f_{k}\left(e^{i \lambda \cdot \Delta I_{k}(x)}-1\right) \mid \mathscr{F}_{s}\right\} \\
&=E\left\{f_{k}\left[i \lambda \cdot \Delta I_{k}(x)+R_{2}\left(\lambda \cdot \Delta I_{k}(x)\right)\right] \mid \mathscr{F}_{s}\right\} \\
&=E\left\{f_{k} e^{-i \lambda \cdot \Delta w\left(t_{k}\right)} e^{i \lambda \cdot \Delta w\left(t_{k}\right)} i \lambda \cdot \Delta I_{k}(x) \mid \mathscr{F}_{s}\right\} \\
&+E\left\{f_{k} R_{2}\left(\lambda \cdot \Delta I_{k}(x)\right) \mid \mathscr{F}_{s}\right\} \\
&=E\left\{f_{k} e^{-i \lambda \cdot \Delta w\left(t_{k}\right)}\left[1+R_{1}\left(\lambda \cdot \Delta w\left(t_{k}\right)\right)\right] i \lambda \cdot \Delta I_{k}(x) \mid \mathscr{F}_{s}\right\} \\
&+E\left\{f_{k} e^{-i \lambda \cdot \Delta w\left(t_{k}\right)} e^{i \lambda \cdot \Delta w\left(t_{k}\right)} R_{2}\left(\lambda \cdot \Delta I_{k}(x)\right) \mid \mathscr{F}_{s}\right\} \\
&= E\left\{f_{k} e^{-i \lambda \cdot \Delta w\left(t_{k}\right)}\left[i \lambda \cdot \Delta I_{k}(x)+\left(J_{1}+J_{2}\right)(k)\right] \mid \mathscr{F}_{s}\right\},
\end{aligned}
$$

where

$$
\begin{aligned}
& J_{1}(k)=e^{i \lambda \cdot \Delta w\left(t_{k}\right)} R_{2}\left(\lambda \cdot \Delta I_{k}(x)\right), \\
& J_{2}(k)=i \lambda \cdot \Delta I_{k}(x) R_{1}\left(\lambda \cdot \Delta w\left(t_{k}\right)\right) .
\end{aligned}
$$

By using the fact that $\mathscr{F}_{t} \subset \mathcal{G}_{t}$ and assumption (1.2),

$$
\begin{aligned}
E\left\{f_{k} e^{\left.-i \lambda \cdot \Delta w\left(t_{k}\right) \lambda \cdot \Delta I_{k}(x) \mid \mathscr{F}_{s}\right\}}\right. & \\
& =E\left\{E\left(e^{i \lambda \cdot\left[w(t)-w\left(t_{k+1}\right)+v\left(t_{k}\right)-v(s)\right]}\left[\lambda \cdot \Delta I_{k}(x)\right] \mid \mathcal{G}_{t_{k+1}}\right) \mid \mathscr{F}_{s}\right\} \\
& =E e^{i \lambda \cdot\left[w(t)-w\left(t_{k+1}\right)\right]} E\left\{e^{i \lambda \cdot\left[v\left(t_{k}\right)-v(s)\right]}\left[\lambda \cdot \Delta I_{k}(x)\right] \mid \mathscr{F}_{s}\right\} \\
& =E e^{i \lambda \cdot\left[w(t)-w\left(t_{k+1}\right)\right]} E\left\{e^{i \lambda \cdot\left[v\left(t_{k}\right)-v(s)\right]} E\left(\lambda \cdot \Delta I_{k}(x) \mid \mathscr{F}_{t_{k}}\right) \mid \mathscr{F}_{s}\right\} \\
& =0 .
\end{aligned}
$$

The last inequality follows from the facts that $\Delta I_{k}(x)=\Delta v\left(t_{k}\right)-$ $\Delta w\left(t_{k}\right),\left\{v(t), \mathscr{F}_{t}\right\}$ is a martingale, and assumptions (1.2) and (2.1). Therefore, (2.8) and (2.10) imply equality (2.4). Inequality (2.5) follows from (2.9), (2.7) 
and the fact that $\left|e^{i \lambda \cdot w\left(t_{k}\right)}\right|=1$. This completes the proof of Lemma 2.

Theorem 3. For $0 \leqslant s<t \leqslant T$,

$$
E\left\{e^{i \lambda \cdot[v(t)-v(s)]} \mid \mathscr{F}_{s}\right\}=E e^{i \lambda \cdot[w(t)-w(s)]} .
$$

Proof. By Lemma 2,

$$
\begin{aligned}
E\left\{e^{i \lambda \cdot[v(t)-v(s)]}-e^{i \lambda \cdot[w(t)-w(s)]} \mid \mathscr{F}_{s}\right\} \\
=E\left(f_{2^{n}}-f_{0} \mid \mathscr{F}_{s}\right)=\sum_{k} E\left(f_{k+1}-f_{k} \mid \mathscr{F}_{s}\right) \\
=\sum_{k} E\left\{f_{k} e^{-i \lambda \cdot \Delta w\left(t_{k}\right)}\left(J_{1}+J_{2}\right)(k) \mid \mathscr{F}_{s}\right\}
\end{aligned}
$$

From the fact that $\left|f_{k} e^{-i \lambda \cdot \Delta w\left(t_{k}\right)}\right| \leqslant 1$, and from Lemma 1 , it is easily seen that

$$
\lim _{n \rightarrow \infty} \sum_{k} E\left|f_{k} e^{-i \lambda \cdot \Delta w\left(t_{k}\right)}\left(J_{1}+J_{2}\right)(k)\right|=0 .
$$

Therefore, by letting $n \rightarrow \infty$ in (2.11), we have

$$
E\left\{e^{i \lambda \cdot[v(t)-v(s)]}-e^{i \lambda \cdot[w(t)-w(s)]} \mid \mathscr{F}_{s}\right\}=0 .
$$

Since $\mathscr{F}_{s} \subset \mathcal{G}_{s}$, Theorem 3 follows from the above identity and from assumption (1.2).

Corollary 4. The innovation process $\left\{v(t), \mathscr{F}_{t}\right\}, 0<t<T$, is a process of independent increments with the same probability law as $w(t)$.

ACKNOWLEDgement. The author is indebted to the referee for his valuable comments.

\section{BIBLIOGRAPHY}

1. A. Segall and T. Kailath, The modeling of randomly modulated jump processes, IEEE Trans. Information Theory IT-21 (1975), 135-143. MR 51 \# 2731.

2. P. A. Frost, Estimation and detection for a simple class of conditionally independent increment processes, Proc. IEEE Decision and Control Conf., 1971.

3. M. Fujisaki, G. Kallianpur and H. Kunita, Stochastic differential equations for the nonlinear filtering problem, Osaka J. Math. 9 (1972), 19-40. MR 49 \#1574.

4. T. Kailath, An innovations approach to least-square estimation. Part I, IEEE Trans. Automatic Control AC = 13 (1968), 645-655; comment, ibid. AC-15 (1970), 158-159. MR 46 \#8367.

5. __ Some extensions of the innovation theorem, Bell System Tech. J. 50 (1971), $1487-1494$.

6. $\quad$ A note on least square estimation by the innovation method, SIAM J. Control 10 (1972), 477-486.

7. A. Lindquist, Optimal filtering of continuous-time stationary processes by means of the backward innovation process, SIAM J. Control 12 (1974), 747-754.

Department of Mathematics, Louisiana State University, Baton Rouge, louisiana 70803

Current address: Department of Business Mathematics, Soochow University, Taipei, Taiwan, Republic of China 